\title{
Use of microalgae: tetraselmis tetrathele extract in formulation of nanoemulsions for cosmeceutical application
}

\begin{abstract}
Cosmeceuticals are cosmetic-hybrids intended to enhance health and beauty of the skin. Topical delivery of antioxidants from natural bioresources has gained attention in line with the increasing demand for harmless cosmetics. Tetraselmis tetrathele is a microalgae species, which has high antioxidant contents. In this work, ternary phase analysis with different compositions of oil, surfactant, and water was conducted to evaluate homogeneity and stability of nanoemulsion lotion containing $1 \% \mathrm{~T}$. tetrathele extract. The three formulations $\mathrm{T} 1, \mathrm{~T} 2$, and $\mathrm{T} 3$ containing various percentages of the surfactant Tween 80 , T1 (20 wt $\%$ of Tween 80), T2 (15 wt $\%$ of Tween 80 ), and T3 (10 wt $\%$ of Tween 80$)$, were analyzed for size and zeta potential to evaluate stability of the nanoemulsion. All particles were nanosized ranging from 102.3 to $249.5 \mathrm{~nm}$. Zeta potential analysis for all emulsions showed negative values from -33.2 to $-71.7 \mathrm{mV}$, which indicates high stability of the nanoemulsion. In order to evaluate the storage stability, a stability test was conducted at different temperature levels $\left(4,25\right.$, and $\left.45{ }^{\circ} \mathrm{C}\right)$ for 10 weeks. At all temperature conditions, T1, T2, and T3 were stable with exception of T3 that precipitated and sedimented after 8 weeks. This study illustrated that $\mathrm{T}$. tetrathele extract can be used as bioactive compound for nanocosmeceutical products, which has high homogeneity and stability.
\end{abstract}

Keyword: Topical nanoemulsions; Microalgae; Tetraselmis tetrathele extract 